• 研究报告・

\title{
极小种群野生植物梓叶槭的种实表型变异特征
}

\author{
冯秋红 ${ }^{1,2}$ 李登峰 1,4 于 涛 $^{3}$ 李俊清 $^{3}$ 马文宝 ${ }^{1,2}$ 张 雷 $^{5}$ \\ 1 (四川省林业科学研究院, 四川卧龙森林生态系统定位站, 成都 610081) \\ 2 (森林和湿地生态恢复与保育四川重点实验室, 成都 610081) \\ 3 (北京林业大学省部共建森林培育与保护教育部重点实验室, 北京 100083) \\ 4 (四川农业大学, 成都 611130) \\ 5 (国家林业和草原局森林培育重点实验室, 中国林业科学研究院林业研究所, 北京 100091)
}

摘要: 表型变异是植物遗传变异与环境异质性相互作用的结果, 是植物适应和进化的具体表现。为揭示极小种群 植物梓叶槭(Acer catalpifolium)的种实表型在其适应环境过程中的变异特征, 明确梓叶槭优质种源地, 为该物种的 保护提供基础数据, 本研究以天然分布的 5 个小种群的 11 个种实性状为研究对象, 采用巢式方差、变异系数、表型 分化系数等多种指标, 探讨了其种群间和种群内的表型变异。结果表明: 梓叶棫种实表型性状在种群间和种群内 均存在着显著的差异, 种群内的变异(63.11\%)大于种群间的变异(23.61\%), 种群间平均分化系数为 $27.23 \%$, 分化 程度在械树科植物中相对较大。种子厚度的平均变异系数最高(40.64\%), 其次是果柄长。翅果千粒重的平均变异 系数最小 (1.57\%), 是最稳定的种实性状。都江堰种群的表型多样性最丰富, 平武种群的则最低; 气象因子对梓叶 槭种实表型性状的影响不显著。就种实表型性状而言, 虽然雷波种群的翅果最大, 平武种群的种子最大, 同时两者 均具有最大的种子厚度和质量均一度。故平武和雷波种群均可作为梓叶槭优质种质资源的候选地。

关键词: 梓叶槭; 种子; 翅果; 表型性状; 气象因子; 极小种群

\section{Phenotypic fruit and seed variations of Acer catalpifolium, a Wild Plant with Extremely Small Populations in China}

Qiuhong Feng ${ }^{1,2^{*}}$, Dengfeng $\mathrm{Li}^{1,4}$, Tao $\mathrm{Yu}^{3}$, Junqing $\mathrm{Li}^{3}$, Wenbao Ma ${ }^{1,2}$, Lei Zhang ${ }^{5}$

1 Sichuan Academy of Forestry, Sichuan Wolong Forest Ecosystem Research Station, Chengdu 610081

2 Ecological Restoration and Conservation on Forest and Wetland Key Laboratory of Sichuan Province, Chengdu 610081

3 Key Laboratory for Silviculture and Conservation of MOE, Beijing 100083

4 Sichuan Agricultural University, Chengdu 611130

5 Key Laboratory of Forest Silviculture of the State Forestry and Grassland Administration, Research Institute of Forestry, Chinese Academy of Forestry, Beijing 100091

Abstract: Phenotypic variation can indicate the adaptation of plant species to different environmental conditions due to long-term evolution. Acer catalpifolium is a relatively primitive species in Aceraceae family, and is a rare and endangered species in China. To understand the range of phenotypic variation of $A$. catalpifolium's fruits and seeds, 11 traits for 47 individuals from five natural small populations of $A$. catalpifolium were measured and parameterized using coefficients of variance, variation, and correlation. The results showed significant differences in phenotypic variation among and within populations. Overall variation was greater within population $(63.11 \%)$ than among populations $(23.61 \%)$ while the mean phenotypic differentiation coefficient was $27.23 \%$ among populations, larger than other species in Aceraceae. The average variation coefficient of seed thickness (ST) was the highest (40.64\%), followed by fruit stalk length (FSL); thousand-fruit weight (TFW) was the smallest and the most stable phenotypic trait $(1.57 \%)$. The Dujiangyan population has the highest phenotypic diversity and Pingwu population the lowest. Meteorological factors were not associated to fruit and seed size variations of A. catalpifolium. The fruits of 
the Leibo population were largest while the seeds of Pingwu population were the biggest. These two populations also have the thickest seeds. Fruit/seed size is considered as an indicator of quality, because bigger fruit/seed could provide more nutrients for next generations, therefore, the Leibo and Pingwu populations should be the priority when collecting germplasms of A. catalpifolium.

Key words: Acer catalpifolium; seed and fruit; phenotypic traits; meteorological factors; extremely small populations

梓叶槭(Acer catalpifolium)为槭树科槭属落叶 大乔木, 是我国特有的珍稀濒危树种, 国家II级保 护植物。其树形高大优美, 材质坚硬、致密, 不但 是优良的用材树种, 也可作为绿化观赏树种。该树 种主要产于四川省, 常零星分布于400-2,000 m的 常绿阔叶林中。因长期的人为干扰和自身天然更新 困难(马文宝等, 2014), 根据前人调查, 梓叶槭种群 数量极为有限, 已处于极度濒危状态(方文培, 1981; 余道平等, 2008), 属极小种群野生植物(Ren et al, 2012; 国政和威润国, 2013)。

植物的表型是由遗传因素和环境因素共同决 定的。表型变异是同种植物适应不同环境条件的结 果, 所以表型变异在适应和进化上具有重要意义 (李斌等, 2002)。果实和种子是植物的生殖器官, 是 植物种群生殖生态学的重要研究对象。果实和种子 的表型性状不仅决定了物种的扩散方式和能力, 也 影响到种子的萌发和幼苗的定居, 进而影响到种群 的分布格局(李帅锋等, 2013)。植物在不同的分布区 域适应不同的环境条件, 种实表型因而发生分化, 种子的形态学性状的地带性变化规律已成为种子 地理学研究的内容之一(于顺利和方伟伟, 2012)。国 内外对松科、柏科等裸子植物种实表型多样性的研 究较多, 针对茶条槭(Acer ginnala)、葛夢槭(A. grosseri)、金钱槭(Dipteronia sinensis)等槭树科植物的 表型多样性亦均有了一定的研究(李珊等, 2003; 王 丹等, 2010; 孟超等, 2013), 但针对梓叶槭种实多样
性的研究还未见报道。梓叶槭属于槭树科槭属较为 原始的种类, 与其他槭树科植物相比, 不但现存数 量极少, 而且分布范围有限, 仅在四川境内有分 布。通过对梓叶槭分布范围内天然种群种实表型特 征研究, 不但可以了解该物种种实表型变异特征及 其对环境因子的响应, 也可为槭属植物系统进化和 环境适应等研究提供基础数据。

本文以梓叶槭的 5 个天然小种群为研究对象, 测定了翅果和种子的 11 个性状, 旨在揭示梓叶槭种 实的表型变异程度与变异规律, 充实我国特有珍稀 濒危植物梓叶槭生物学特性基本数据库、促进该物 种的种质资源保存与优质种质资源的选育, 并为其 保护与繁育研究提供参考。

\section{材料与方法}

\section{1 研究区域概况}

研究地点位于有梓叶槭分布的四川省都江堰、 平武、雷波、大邑、峨眉山共 5 个区域。研究地点 的地理和环境信息详见表1。

\section{2 研究方法}

\subsection{1 样地设置与调查}

2016年11-12月在梓叶棫的天然分布区内, 选择 上述5个分布区的小种群, 每个分布点选择6-16株成 年母树进行翅果和种子的采集工作, 每个植株采集 20 粒翅果和种子。单株选择标准为植株生长良好、 无严重缺陷且无病虫害。采样点地理生态因子(四川

表1 研究地点的地理及环境信息

Table 1 Geographical and environmental information of the five natural small populations of Acer catalpifolium

\begin{tabular}{|c|c|c|c|c|}
\hline $\begin{array}{l}\text { 研究地点 } \\
\text { Site }\end{array}$ & $\begin{array}{l}\text { 经纬度 } \\
\text { Latitude and longitude }\end{array}$ & $\begin{array}{l}\text { 海拔 } \\
\text { Altitude (m) }\end{array}$ & $\begin{array}{l}\text { 年平均气温 } \\
\text { Mean annual temperature }\left({ }^{\circ} \mathrm{C}\right)\end{array}$ & $\begin{array}{l}\text { 年平均降水量 } \\
\text { Mean annual precipitation (mm) }\end{array}$ \\
\hline 都江堰 Dujiangyan & $\begin{array}{l}103^{\circ} 31^{\prime} 28^{\prime \prime}-103^{\circ} 32^{\prime} 50^{\prime \prime} \mathrm{E}, \\
30^{\circ} 51^{\prime} 22^{\prime \prime}-30^{\circ} 53^{\prime} 27^{\prime \prime} \mathrm{N}\end{array}$ & 823 & 14.28 & 1,155 \\
\hline 平武 Pingwu & $104^{\circ} 23^{\prime} 41^{\prime \prime} \mathrm{E}, 32^{\circ} 14^{\prime} 14^{\prime \prime} \mathrm{N}$ & 1,120 & 15.20 & 957 \\
\hline 雷波 Leibo & $\begin{array}{l}103^{\circ} 39^{\prime} 50^{\prime \prime}-103^{\circ} 39^{\prime} 56^{\prime \prime} \mathrm{E}, \\
28^{\circ} 31^{\prime} 13^{\prime \prime}-28^{\circ} 31^{\prime} 24^{\prime \prime} \mathrm{N}\end{array}$ & 995 & 14.50 & 1,099 \\
\hline 大邑 Dayi & $\begin{array}{l}103^{\circ} 15^{\prime} 36^{\prime \prime}-103^{\circ} 22^{\prime} 28^{\prime \prime} \mathrm{E}, \\
30^{\circ} 36^{\prime} 08^{\prime \prime}-30^{\circ} 42^{\prime} 02^{\prime \prime} \mathrm{N}\end{array}$ & 1,960 & 15.35 & 1,223 \\
\hline 峨眉山 Emeishan & $\begin{array}{l}103^{\circ} 24^{\prime} 04^{\prime \prime}-103^{\circ} 26^{\prime} 18^{\prime \prime} \mathrm{E}, \\
29^{\circ} 33^{\prime} 45^{\prime \prime}-29^{\circ} 35^{\prime} 02^{\prime \prime} \mathrm{N}\end{array}$ & 718 & 16.07 & 1,376 \\
\hline
\end{tabular}


表2 采样地点地形地貌以及调查母树信息表

Table 2 Geomorphic information of sites and investigated mother trees for the five natural small populations of Acer catalpifolium

\begin{tabular}{|c|c|c|c|c|c|c|c|}
\hline \multirow{2}{*}{$\begin{array}{l}\text { 种群 } \\
\text { Population }\end{array}$} & \multirow{2}{*}{$\begin{array}{l}\text { 坡度 } \\
\text { Slope } \\
\left(^{\circ}\right)\end{array}$} & \multirow{2}{*}{$\begin{array}{l}\text { 坡向 } \\
\text { Aspect }\end{array}$} & \multirow{2}{*}{$\begin{array}{l}\text { 地貌类型 } \\
\text { Geomorphic type }\end{array}$} & \multicolumn{4}{|c|}{ 调查母树 Mother tree investigated } \\
\hline & & & & $\begin{array}{l}\text { 调查数量 } \\
\text { Number }\end{array}$ & $\begin{array}{l}\text { 平均树高 } \\
\text { Mean height (m) }\end{array}$ & $\begin{array}{l}\text { 平均胸径 } \\
\text { Mean DBH (cm) }\end{array}$ & $\begin{array}{l}\text { 平均冠幅 } \\
\text { Mean crown }\end{array}$ \\
\hline $\begin{array}{l}\text { 都江堰 } \\
\text { Dujiangyan }\end{array}$ & 15 & $\begin{array}{l}\text { 南、东南、东北、西北 } \\
\text { South, southeast, northeast, } \\
\text { northwest }\end{array}$ & 山地 Mountain & 16 & 14 & 15 & $7 \mathrm{~m} \times 7 \mathrm{~m}$ \\
\hline $\begin{array}{l}\text { 平武 } \\
\text { Pingwu }\end{array}$ & 40 & $\begin{array}{l}\text { 西南/北 } \\
\text { Southwest, north }\end{array}$ & $\begin{array}{l}\text { 沟边山地 } \\
\text { Valley side mountain }\end{array}$ & 6 & 15.5 & 34 & $7 \mathrm{~m} \times 7 \mathrm{~m}$ \\
\hline 雷波 Leibo & 20 & 东南 Southeast & 山地 Mountain & 9 & 7 & 12 & $5 \mathrm{~m} \times 4 \mathrm{~m}$ \\
\hline $\begin{array}{l}\text { 大邑 } \\
\text { Dayi }\end{array}$ & 38 & $\begin{array}{l}\text { 南、北 } \\
\text { South, north }\end{array}$ & 山地 Mountain & 6 & 9.5 & 20 & $6 \mathrm{~m} \times 7 \mathrm{~m}$ \\
\hline $\begin{array}{l}\text { 峨眉山 } \\
\text { Emeishan }\end{array}$ & 15 & $\begin{array}{l}\text { 北、西南、南 } \\
\text { North, southwest, south }\end{array}$ & 山地 Mountain & 10 & 13 & 23 & $12 \mathrm{~m} \times 12 \mathrm{~m}$ \\
\hline
\end{tabular}

植被协作组, 1980)、母树生长情况见表1和表2。

\subsection{2 梓叶槭种实形态指标的测定}

选择易于获取的 9 个种实表型性状进行测定。具 体包括: 翅果长(KFL)、翅果宽 (KFW)、翅果长宽比 $(\mathrm{KFLW}) 、$ 着生痕 $(\mathrm{BM}) 、$ 种子长 $(\mathrm{SL}) 、$ 种子宽 $(\mathrm{SW}) 、$ 种子长宽比(SLW)、种子厚度(ST)和果柄长(FSL)。 用游标卡尺分别测量其长宽(详见图1), 测量精度为 $0.01 \mathrm{~mm}$ 。此外, 以每个采集点为单位, 收集样株翅 果(手动去皮获得种子), 每株500粒, 混匀后利用万 分位天平称重, 再换算成翅果/种子千粒重, 重复 3次。

\section{3 数据统计分析}

使用Climate-China (V4.4)对1971-2000年的 PRISM数据进行降尺度处理, 提取各采样点的地理 坐标和海拔的气象信息(Daly et al, 2002; 张雷等, 2011), 选择气象指标包括平均温度、年平均降水

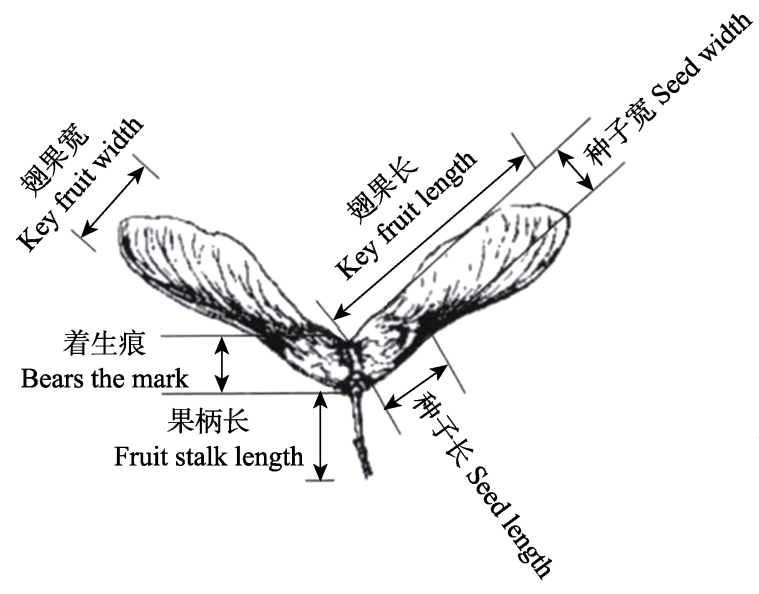

图1 梓叶槭果实形态示意图

Fig. 1 Sketch map of six fruit traits of Acer catalpifolium
量、年平均最暖月温度、年平均最冷月温度和年平 均生长季(5-9月)降水量等，以期揭示梓叶槭种实对 于水热等主要气象因子的响应和适应。

计算获取各表型性状的平均值、标准差以及变 异系数(CV); 在对所有数据进行齐性检验的基础上, 开展表型性状间的差异性检验和LSD或Duncan多 重比较分析; 利用巢式设计的方差分析法(葛颂等, 1988)对各种群不同单株的表型性状值进行分析; 利 用方差分析的结果计算表型分化系数(公式1), 该指 标是描述性状种群间平均方差占总方差的比例，表 明表型变异在种群间的贡献大小(冯秋红等, 2017)。

$$
\text { 表型分化系数 }=\frac{\text { 种群间方差值 }}{\text { 种群间方差值种群内方差值 }}
$$

同时，还对梓叶槭种实9种表型性状间以及种 实11种性状与主要气象因子间的相关关系进行了 Pearson相关分析。因为与其他 9 种表型性状不同，果 实千粒重与种子千粒重的取样单位为种群, 故仅对 其进行了性状平均值、标准差、变异系数和差异性 检验分析。

齐性检验、方差分析相关分析通过SPSS 16.0 完成, 其他统计分析按照常规方法并利用EXCEL 2010进行计算。

\section{结果}

\section{1 梓叶槭天然种群种实表型性状及其形态变异} 特征

种实的11种性状的平均值、标准偏差和多重比 较结果见表3。梓叶槭种实性状间存在着显著差异。 
雷波种群具有最大的翅果长、翅果宽、种子厚度、 翅果千粒重, 但是最小的果柄长。平武种群具有最 大的着生痕、种子长、种子宽、种子厚度、翅果长 宽比、种子千粒重，但最小的翅果宽和种子长宽比。 大邑种群具有最大的果柄长和种子长宽比, 但翅果 长、着生痕和种子厚度最小。都江堰种群具有最小的 种子长、种子宽、种子厚度、翅果长宽比、翅果和 种子千粒重。峨眉山种群具有最小的翅果长宽比。

变异系数是表型性状离散特征的表现。变异系 数越大, 性状的离散程度越大, 表型多样性越丰富; 反之则表型多样性越贫乏。梓叶槭5个小种群种实 性状的变异系数详见表4。梓叶槭各性状平均变异 系数为 $15.03 \%$, 变异幅度为 $1.57 \%-40.64 \%$ 。11个性 状的平均变异系数具有一定的差异: 其中种子厚度 最大, 其次是果柄长, 翅果千粒重最小。可见, 翅果 千粒重的稳定性最高, 而种子厚度的稳定性最差。5 个种群所有性状的平均变异均介于 $14.29 \%-16.23 \%$ 之间, 都江堰种群的表型多样性最为丰富,平武种 群的表型多样性最低。

\section{2 梓叶槭天然种群种实表型变异和分化特征}

梓叶槭种实表型性状在种群间和种群内的差 异分析见表5。经 $F$ 检验, 梓叶槭的翅果和种子的 9 个表型性状在种群间和种群内均存在极显著差异 $(P$ $<0.01$ )。

根据巢式方差分析结果(表6), 9个表型性状在 种群间和种群内的平均方差分量百分比分别是
23.61\%和63.11\%, 具有一定的差异。表型分化系数 的变异范围为 $2.41 \%-46.60 \%$, 着生痕的表型分化系 数最大 $(46.60 \%)$, 其次是果柄长 $(46.48 \%)$ 和种子宽 (35.25\%), 翅果长宽比的分化系数最小 $(2.41 \%)$ 。种 群间平均表型分化系数为 $23.61 \%$, 说明梓叶槭小种 群种实表型变异在种群间的贡献仅占 $23.61 \%$, 种群 内的变异更大，种群内多样性高于种群间。

\section{3 梓叶槭种实表型性状间的相关关系}

梓叶槭各种实表型性状间的相关关系见表7。 首先，除种子长和着生痕之间无显著相关外，翅果 长、翅果宽、着生痕、种子长、种子宽和种子厚度 6 个性状两两之间均呈现极显著正相关。其次, 果柄 长与翅果长、翅果宽和着生痕之间呈现极显著负相 关，而与种子长呈极显著正相关，与种子宽和种子 厚度无显著相关。翅果长宽比除了与翅果长呈极显 著正相关、与翅果宽呈极显著负相关外，与其他性 状间均无显著相关。最后, 种子长宽比与着生痕、 种子宽分别呈现极显著负相关，而与种子长、种子 厚度和果柄长呈现极显著正相关。

梓叶槭种子和翅果千粒重与表型性状间的相 关关系详见表 8 。首先，9个种实表型性状均与种子 和翅果千粒重呈现正相关; 其次, 种子长、种子宽、 种子厚度与种子和翅果千粒重均呈现极显著正相 关，而翅果长与翅果千粒重呈极显著正相关，与种 子千粒重呈现显著正相关，翅果宽则仅与翅果千粒 重呈现显著正相关，其余种实表型性状与种子和翅

表3 梓叶槭天然小种群间种实性状的平均值及标准偏差

Table 3 Means and standard deviations of 11 fruit traits in the five natural small populations of Acer catalpifolium

\begin{tabular}{|c|c|c|c|c|c|c|c|c|c|c|c|c|}
\hline \multirow{2}{*}{$\begin{array}{l}\text { 种群 } \\
\text { Population }\end{array}$} & \multirow{2}{*}{$\begin{array}{l}\text { 样本 } \\
\text { 量 } \\
\text { Sample } \\
\text { size }\end{array}$} & \multicolumn{11}{|c|}{ 平均值土标准差 Mean \pm SD } \\
\hline & & $\begin{array}{l}\text { 翅果长 } \\
\mathrm{KFL} \\
(\mathrm{cm})\end{array}$ & $\begin{array}{l}\text { 翅果宽 } \\
\text { KFW } \\
(\mathrm{cm})\end{array}$ & $\begin{array}{l}\text { 着生痕 } \\
\mathrm{BM}(\mathrm{cm})\end{array}$ & $\begin{array}{l}\text { 种子长 } \\
\text { SL (cm) }\end{array}$ & $\begin{array}{l}\text { 种子宽 } \\
\text { SW }(\mathrm{cm})\end{array}$ & $\begin{array}{l}\text { 种子厚度 } \\
\text { ST (cm) }\end{array}$ & $\begin{array}{l}\text { 果柄长 } \\
\text { FSL (cm) }\end{array}$ & $\begin{array}{l}\text { 翅果 } \\
\text { 长宽比 } \\
\text { KFLW }\end{array}$ & $\begin{array}{l}\text { 种子 } \\
\text { 长宽比 } \\
\text { SLW }\end{array}$ & $\begin{array}{l}\text { 翅果 } \\
\text { 千粒重 } \\
\text { TFW (g) }\end{array}$ & $\begin{array}{l}\text { 种子 } \\
\text { 千粒重 } \\
\text { TSW (g) }\end{array}$ \\
\hline $\begin{array}{l}\text { 都江堰 } \\
\text { Dujiangyan }\end{array}$ & 320 & $3.24 \pm 0.35^{c}$ & $1.11 \pm 0.15^{\mathrm{c}}$ & $0.54 \pm 0.05^{\mathrm{b}}$ & $0.77 \pm 0.12^{\mathrm{d}}$ & $0.46 \pm 0.06^{\mathrm{e}}$ & $0.11 \pm 0.04^{\mathrm{c}}$ & $1.42 \pm 0.39^{\mathrm{d}}$ & $2.93 \pm 0.32^{\mathrm{ab}}$ & $1.71 \pm 0.23^{b}$ & $2.21 \pm 0.03^{c}$ & $0.84 \pm 0.03^{\mathrm{c}}$ \\
\hline $\begin{array}{l}\text { 平武 } \\
\text { Pingwu }\end{array}$ & 120 & $3.38 \pm 0.27^{b}$ & $1.10 \pm 0.09^{c}$ & $0.68 \pm 0.05^{\mathrm{a}}$ & $0.92 \pm 0.12^{\mathrm{a}}$ & $0.56 \pm 0.05^{\mathrm{a}}$ & $0.13 \pm 0.09^{\mathrm{a}}$ & $2.10 \pm 0.49^{b}$ & $3.07 \pm 0.21^{\mathrm{ab}}$ & $1.63 \pm 0.17^{c}$ & $3.03 \pm 0.03^{\mathrm{a}}$ & $1.40 \pm 0.05^{\mathrm{a}}$ \\
\hline $\begin{array}{l}\text { 雷波 } \\
\text { Leibo }\end{array}$ & 180 & $3.71 \pm 0.26^{\mathrm{a}}$ & $1.22 \pm 0.10^{\mathrm{a}}$ & $0.49 \pm 0.04^{\mathrm{c}}$ & $0.89 \pm 0.12^{\mathrm{ab}}$ & $0.52 \pm 0.06^{\mathrm{b}}$ & $0.13 \pm 0.03^{\mathrm{a}}$ & $1.24 \pm 0.29^{\mathrm{e}}$ & $3.06 \pm 0.27^{\mathrm{a}}$ & $1.76 \pm 0.41^{\mathrm{b}}$ & $3.41 \pm 0.05^{\mathrm{a}}$ & $1.38 \pm 0.02^{\mathrm{a}}$ \\
\hline $\begin{array}{l}\text { 大邑 } \\
\text { Dayi }\end{array}$ & 120 & $3.21 \pm 0.32^{\mathrm{d}}$ & $1.11 \pm 0.15^{c}$ & $0.48 \pm 0.06^{\mathrm{d}}$ & $0.86 \pm 0.14^{b}$ & $0.49 \pm 0.05^{d}$ & $0.11 \pm 0.03^{\mathrm{c}}$ & $2.21 \pm 0.65^{\mathrm{a}}$ & $3.01 \pm 1.11^{\mathrm{ab}}$ & $1.78 \pm 0.27^{\mathrm{a}}$ & $2.73 \pm 0.07^{\mathrm{b}}$ & $1.08 \pm 0.05^{\mathrm{b}}$ \\
\hline $\begin{array}{l}\text { 峨眉山 } \\
\text { Emeishan }\end{array}$ & 200 & $3.41 \pm 0.32^{\mathrm{b}}$ & $1.16 \pm 0.11^{\mathrm{b}}$ & $0.54 \pm 0.04^{\mathrm{b}}$ & $0.84 \pm 0.1^{\mathrm{c}}$ & $0.50 \pm 0.05^{\mathrm{c}}$ & $0.12 \pm 0.02^{\mathrm{b}}$ & $1.59 \pm 0.40^{c}$ & $2.93 \pm 0.25^{\mathrm{b}}$ & $1.71 \pm 0.23^{\mathrm{b}}$ & $2.86 \pm 0.04^{b}$ & $1.01 \pm 0.03^{\mathrm{b}}$ \\
\hline
\end{tabular}

不同小写字母表示同一列数据间差异显著 $(P<0.05)$

Different small letters mean significant differences $(P<0.05)$. KFL, Key fruit length; KFW, Key fruit width; KFLW, The ratio of key fruit length to width; BM, Bears the mark; SL, Seed length; SW, Seed width; SLW, The ratio of seed length to seed width; SWSC, Seed weight in single key fruit; ST, Seed thickness; FSL, Fruit stalk length; TFW, Thousand-fruit weight; TSW, Thousand-seed weight. 
表4＼cjkstart梓叶槭天然小种群表型性状的变异系数(性状代号同表3)

Table 4 Variation coefficients of phenotypic traits in Acer catalpifolium natural small populations. The phenotypic traits are the same as Table 3.

\begin{tabular}{|c|c|c|c|c|c|c|}
\hline \multirow{2}{*}{$\begin{array}{l}\text { 表型状性 } \\
\text { Phenotypic trait }\end{array}$} & \multicolumn{5}{|c|}{ 变异系数 Variation coefficients (\%) } & \multirow{2}{*}{$\begin{array}{l}\text { 平均值 } \\
\text {-Mean (\%) }\end{array}$} \\
\hline & $\begin{array}{l}\text { 都江堰种群 } \\
\text { Dujiangyan }\end{array}$ & $\begin{array}{l}\text { 平武种群 } \\
\text { Pingwu }\end{array}$ & $\begin{array}{l}\text { 雷波种群 } \\
\text { Leibo }\end{array}$ & $\begin{array}{l}\text { 大邑种群 } \\
\text { Dayi }\end{array}$ & $\begin{array}{l}\text { 峨眉山种群 } \\
\text { Emeishan }\end{array}$ & \\
\hline 翅果长 KFL & 14.68 & 7.87 & 6.84 & 11.18 & 17.09 & 11.53 \\
\hline 翅果宽 KFW & 14.8 & 8.00 & 8.77 & 11.1 & 11.87 & 10.91 \\
\hline 着生痕 BM & 9.98 & 6.63 & 13.68 & 22.14 & 8.31 & 12.15 \\
\hline 种子长 SL & 16.76 & 13.17 & 15.7 & 16.21 & 21.12 & 16.59 \\
\hline 种子宽 SW & 14.99 & 8.35 & 10.91 & 11.09 & 14.69 & 12.01 \\
\hline 种子厚度 ST & 46.16 & 67.73 & 39.74 & 22.7 & 26.86 & 40.64 \\
\hline 果柄长 FSL & 28.9 & 23.57 & 25.88 & 30.13 & 34.16 & 28.53 \\
\hline 翅果长宽比 KFLW & 13.71 & 6.86 & 9.95 & 10.89 & 11.59 & 10.6 \\
\hline 种子长宽比 SLW & 13.84 & 10.34 & 28.61 & 19.31 & 15.93 & 17.61 \\
\hline 翅果千粒重 TFW & 1.44 & 0.88 & 1.37 & 2.63 & 1.53 & 1.57 \\
\hline 种子千粒重 TSW & 3.28 & 3.78 & 1.57 & 4.61 & 2.88 & 3.22 \\
\hline 平均值 Mean & 16.23 & 14.29 & 14.82 & 14.73 & 15.09 & 15.03 \\
\hline
\end{tabular}

表5＼cjkstart梓叶槭各分布点间及分布点内翅果、种子表型性状的方差表(性状代号同表3)

Table 5 Variance of phenotypic traits among and within populations of Acer catalpifolium. The phenotypic traits are the same as Table 3.

\begin{tabular}{llllll}
\hline 表型性状 & \multicolumn{3}{l}{ 均方 Mean square } & & \multicolumn{2}{l}{$F$ 值 $F$ value } \\
\cline { 2 - 5 } & $\begin{array}{l}\text { Phenotypic trait } \\
\text { Among populations }\end{array}$ & $\begin{array}{l}\text { 种群内 } \\
\text { Within population }\end{array}$ & $\begin{array}{l}\text { 随机误差 } \\
\text { Random error }\end{array}$ & $\begin{array}{l}\text { 种群间 } \\
\text { Among populations }\end{array}$ & $\begin{array}{l}\text { 种群内 } \\
\text { Within population }\end{array}$ \\
\hline 翅果长 KFL & 8.7860 & 0.2010 & 0.0058 & $43.7730^{* *}$ & $34.3670^{* *}$ \\
翅果宽 KFW & 0.5060 & 0.0190 & 0.0023 & $25.9540^{* *}$ & $8.3510^{* *}$ \\
着生痕 BM & 0.9340 & 0.0040 & 0.0001 & $245.7320^{* *}$ & $53.2950^{* *}$ \\
种子长 SL & 0.6890 & 0.0220 & 0.0012 & $31.8010^{* *}$ & $18.6870^{* *}$ \\
种子宽 SW & 0.2660 & 0.0040 & 0.0002 & $65.3130^{* *}$ & $20.2830^{* *}$ \\
种子厚度 ST & 0.0220 & 0.0020 & 0.0003 & $9.0660^{* *}$ & $7.7230^{* *}$ \\
果柄长 FSL & 31.9300 & 0.2440 & 0.0107 & $130.8680^{* *}$ & $22.7050^{* *}$ \\
翅果长宽比 KFLW & 0.7130 & 0.4630 & 0.1254 & $1.5420^{* *}$ & $3.6910^{* *}$ \\
种子长宽比 SLW & 1.0790 & 0.1050 & 0.0178 & $10.2480^{* *}$ & $5.9060^{* *}$ \\
\hline
\end{tabular}

** $P<0.01$.

果千粒重间关系不显著。

\section{4 梓叶槭种实表型性状与环境因子的相关关系}

环境因子与梓叶槭种实表型性状间的相关关 系见表9。11个种实性状与气象因子间均无显著相 关关系。

\section{3 讨论}

通过对梓叶槭天然分布的 5 个小种群翅果、种 子等种实表型性状的研究发现, 梓叶槭的种实表型
性状在种群间和种群内都存在极显著的差异，而种 内变异占据了更主要的位置(占比63.11\%), 其中起 主要作用的是翅果长宽比、种子长宽比、翅果长和 种子长等。大量研究表明, 物种的表型变异程度往 往与其分布范围的大小成正比关系(Loveless \& Hamrick, 1984; Hamrick \& Godt, 1990; Frankham et al, 2002), 这在本研究中也得到了证实：梓叶槭分 布范围较小, 且常零散分布, 难以形成单一纯林或 群落, 所以其表型变异较小, 平均变异系数为 
表6 梓叶槭种实表型性状的方差分量及种群间表型分化系数(性状代号同表3)

Table 6 Variance component and differentiation coefficient of phenotypic traits among and within Acer catalpifolium natural small populations. The phenotypic traits are the same as Table 3.

\begin{tabular}{|c|c|c|c|c|c|c|c|}
\hline \multirow{2}{*}{$\begin{array}{l}\text { 表型性状 } \\
\text { Phenotypic trait }\end{array}$} & \multicolumn{3}{|c|}{ 方差分量 Variance component } & \multicolumn{3}{|c|}{ 方差分量百分比 Percentage of variance portion (\%) } & \multirow{2}{*}{$\begin{array}{l}\text { 表型分化系数 } \\
\text { Phenotypic } \\
\text { differentiation } \\
\text { coefficient }\end{array}$} \\
\hline & $\begin{array}{l}\text { 种群间 } \\
\text { Among } \\
\text { populations }\end{array}$ & $\begin{array}{l}\text { 种群内 } \\
\text { Within } \\
\text { population }\end{array}$ & $\begin{array}{l}\text { 随机误差 } \\
\text { Random } \\
\text { errors }\end{array}$ & $\begin{array}{l}\text { 种群间 } \\
\text { Among } \\
\text { populations }\end{array}$ & $\begin{array}{l}\text { 种群内 } \\
\text { Within } \\
\text { population }\end{array}$ & $\begin{array}{l}\text { 随机误差 } \\
\text { Random } \\
\text { errors }\end{array}$ & \\
\hline 翅果长 KFL & 0.0418 & 0.1413 & 0.0066 & 22.06 & 74.48 & 3.46 & 22.85 \\
\hline 翅果宽 KFW & 0.0024 & 0.0053 & 0.0013 & 26.47 & 58.86 & 14.68 & 31.02 \\
\hline 着生痕 BM & 0.0045 & 0.0052 & 0.0013 & 41.28 & 47.29 & 11.43 & 46.60 \\
\hline 种子长 SL & 0.0033 & 0.0108 & 0.0018 & 20.61 & 67.85 & 11.54 & 23.30 \\
\hline 种子宽 SW & 0.0013 & 0.0024 & 0.0009 & 28.54 & 52.43 & 19.03 & 35.25 \\
\hline 种子厚度 ST & 0.0001 & 0.0006 & 0.0004 & 8.23 & 52.10 & 39.67 & 13.64 \\
\hline 果柄长 FSL & 0.1608 & 0.1852 & 0.0078 & 45.46 & 52.35 & 2.20 & 46.48 \\
\hline 翅果长宽比 KFLW & 0.0012 & 0.0495 & 0.0043 & 2.22 & 89.93 & 7.86 & 2.41 \\
\hline 种子长宽比 SLW & 0.0048 & 0.0197 & 0.0026 & 17.68 & 72.71 & 9.61 & 19.56 \\
\hline 平均值 Mean & - & - & - & 23.61 & 63.11 & 13.27 & 27.23 \\
\hline
\end{tabular}

表7 梓叶槭天然小种群 9 个种实表型性状间的相关分析(性状代号同表3)

Table 7 Correlation of 9 fruit traits in Acer catalpifolium natural small populations. The phenotypic traits are same as Table 3.

\begin{tabular}{|c|c|c|c|c|c|c|c|c|c|}
\hline $\begin{array}{l}\text { 表型性状 } \\
\text { Phenotypic trait }\end{array}$ & $\begin{array}{l}\text { 翅果长 } \\
\text { KFL (cm) }\end{array}$ & $\begin{array}{l}\text { 翅果宽 } \\
\text { KFW (cm) }\end{array}$ & $\begin{array}{l}\text { 着生痕 } \\
\mathrm{BM}(\mathrm{cm})\end{array}$ & $\begin{array}{l}\text { 种子长 } \\
\text { SL }(\mathrm{cm})\end{array}$ & $\begin{array}{l}\text { 种子宽 } \\
\text { SW }(\mathrm{cm})\end{array}$ & $\begin{array}{l}\text { 种子厚度 } \\
\text { ST }(\mathrm{cm})\end{array}$ & $\begin{array}{l}\text { 果柄长 } \\
\text { FSL (cm) }\end{array}$ & $\begin{array}{l}\text { 翅果长宽比 } \\
\text { KFLW }\end{array}$ & $\begin{array}{l}\text { 种子长宽比 } \\
\text { SLW }\end{array}$ \\
\hline 翅果长 KFL & 1 & & & & & & & & \\
\hline 翅果宽 KFW & $0.572^{* *}$ & 1 & & & & & & & \\
\hline 着生痕 BM & $0.217^{* *}$ & $0.102^{* *}$ & 1 & & & & & & \\
\hline 种子长 SL & $0.318^{* *}$ & $0.292^{* *}$ & 0.06 & 1 & & & & & \\
\hline 种子宽 SW & $0.417^{* *}$ & $0.372^{* *}$ & $0.151^{* *}$ & $0.541^{* *}$ & 1 & & & & \\
\hline 种子厚度 $\mathrm{ST}$ & $0.121^{* *}$ & $0.089^{* *}$ & $0.116^{* *}$ & $0.418^{* *}$ & $0.251^{* *}$ & 1 & & & \\
\hline 果柄长 FSL & $-0.160^{* *}$ & $-0.084^{*}$ & $-0.179^{* *}$ & $0.154^{* *}$ & 0.040 & 0.063 & 1 & & \\
\hline 翅果长宽比 KFLW & $0.271^{* *}$ & $-0.310^{* *}$ & 0.042 & 0.053 & 0.020 & 0.005 & 0.011 & 1 & \\
\hline 种子长宽比 SLW & -0.016 & 0.000 & $-0.124^{* *}$ & $0.581^{* *}$ & $-0.263^{* *}$ & $0.216^{* *}$ & $0.147^{* *}$ & 0.036 & 1 \\
\hline
\end{tabular}

** $P<0.01$.

表8＼cjkstart梓叶槭果实千粒重与9个种实表型性状间的相关关系(性状代号同表3)

Table 8 Correlation of nine fruit traits and thousand-fruit weigh, thousand-seed weight in the natural small populations of Acer catalpifolium. The phenotypic traits are the same as Table 3.

\begin{tabular}{|c|c|c|c|c|c|c|c|c|c|}
\hline $\begin{array}{l}\text { 表型性状 } \\
\text { Phenotypic trait }\end{array}$ & $\begin{array}{l}\text { 翅果长 } \\
\mathrm{KFL}\end{array}$ & $\begin{array}{l}\text { 翅果宽 } \\
\text { KFW }\end{array}$ & $\begin{array}{l}\text { 着生痕 } \\
\mathrm{BM}\end{array}$ & $\begin{array}{l}\text { 种子长 } \\
\mathrm{SL}\end{array}$ & $\begin{array}{l}\text { 种子宽 } \\
\text { SW }\end{array}$ & $\begin{array}{l}\text { 种子厚度 } \\
\text { ST }\end{array}$ & $\begin{array}{l}\text { 果柄长 } \\
\text { FSL }\end{array}$ & $\begin{array}{l}\text { 翅果长宽比 } \\
\text { KFLW }\end{array}$ & $\begin{array}{l}\text { 种子长宽比 } \\
\text { SLW }\end{array}$ \\
\hline 翅果千粒重 TFW & $0.715^{* *}$ & $0.679^{*}$ & 0.040 & $0.924^{* * *}$ & $0.797^{* *}$ & $0.713^{* *}$ & 0.214 & 0.444 & 0.398 \\
\hline 种子千粒重 TSW & $0.627^{*}$ & 0.478 & 0.145 & $0.919^{* *}$ & $0.766^{* *}$ & $0.796^{* *}$ & 0.270 & 0.494 & 0.434 \\
\hline
\end{tabular}

* $P<0.05$; ** $P<0.01$.

18.88\%, 变异幅度为 $11.38 \%$ - 40.64\%。与其他种群 相比, 平武种群因分布相对较为集中, 故其种实表 型性状的变异系数(表型多样性)也相对较低。正因 为梓叶槭分布范围不大, 其分布区内的温湿度差异 也不大(表1), 所以种群间变异较小。一般来说, 典 型异花授粉的植物, 其交配系统可以促进种群间的
基因交流，增加有效种群大小，减少基因漂变对遗 传结构的影响(Hamrick \& Godt, 1990)。作为槭树科 的高大乔木，梓叶槭虽然是异花授粉植物，但雌雄 同株，这大大促进了其繁衍后代的几率，但同时却 限制了个体间的基因交流，促进了种群内分化。因 为在天然分布的群落中, 近缘种混交情况不常见 
表9 梓叶槭表型性状与气象因子间的相关关系(性状代号同表3)

Table 9 Correlation between phenotypic traits and meteorological factors in Acer catalpifolium natural small populations. The phenotypic traits are same as Table 3.

\begin{tabular}{lllllll}
\hline $\begin{array}{l}\text { 表型性状 } \\
\text { Phenotypic trait }\end{array}$ & $\begin{array}{l}\text { 海拔 } \\
\text { Altitude } \\
(\mathrm{m})\end{array}$ & $\begin{array}{l}\text { 年平均温度 } \\
\text { Mean annual } \\
\text { temperature } \\
\left({ }^{\circ} \mathrm{C}\right)\end{array}$ & $\begin{array}{l}\text { 年平均最暖月温度 } \\
\text { Mean annual } \\
\text { temperature in the } \\
\text { warmest month }\left({ }^{\circ} \mathrm{C}\right)\end{array}$ & $\begin{array}{l}\text { 年平均最冷月温度 } \\
\text { Mean annual } \\
\text { temperature in the } \\
\text { coldest month }\left({ }^{\circ} \mathrm{C}\right)\end{array}$ & $\begin{array}{l}\text { 年平均降水量 } \\
\text { Mean annual } \\
\text { precipitation }(m)\end{array}$ & $\begin{array}{l}\text { 年平均生长季(5-9月) } \\
\text { 降水量 } \\
\text { Mean annual precipitation } \\
\text { between May and } \\
\text { September }(\mathrm{mm})\end{array}$ \\
\hline 翅果长 KFL & 0.124 & 0.184 & 0.099 & 0.266 & 0.124 & -0.019 \\
翅果宽 KFW & 0.145 & 0.182 & 0.101 & 0.226 & 0.384 & 0.191 \\
着生痕 BM & -0.26 & 0.278 & 0.234 & 0.374 & -0.216 & -0.194 \\
种子长 SL & 0.147 & 0.134 & 0.017 & 0.249 & -0.018 & -0.12 \\
种子宽 SW & 0.187 & 0.153 & -0.002 & 0.29 & 0.068 & -0.059 \\
种子厚度 ST & 0.302 & -0.059 & -0.166 & 0.108 & -0.392 & -0.263 \\
果柄长 FSL & -0.265 & 0.165 & 0.161 & 0.174 & -0.04 & -0.016 \\
翅果长宽比 KFLW & 0.013 & 0.138 & 0.098 & 0.192 & -0.122 & -0.208 \\
种子长宽比 SLW & 0.041 & 0.001 & 0.01 & 0.007 & -0.149 & -0.142 \\
翅果千粒重 TFW & 0.264 & 0.145 & 0.000 & 0.289 & -0.003 & -0.082 \\
种子千粒重 TSW & 0.388 & -0.025 & -0.168 & 0.139 & -0.289 & -0.341 \\
\hline
\end{tabular}

(张宇阳等, 2018), 所以基因交流也很难发生。在建 立梓叶槭种质资源圃时, 应该尽量采集不同种源地 的种质资源, 最大程度地保存物种的表型多样性。 表型变异系数指示了植物对于环境的适应潜力, 即 表型变异系数越大, 植物对于环境变化的适应能力 越强, 适应的环境范围可能越广 (李帅锋等, 2013; 冯秋红等, 2017)。梓叶槭平均表型分化系数为 27.33\%，低于云杉(Picea balfouriana) (幸云杰等, 2009)、高山松(Pinus densata) (毛建丰等, 2007)以及 白皮松(P. bungeana) (李斌等, 2002), 但高于思茅松 (P. kesiya) (李帅锋等, 2013)和岷江柏(Cupressus chengiana) (冯秋红等, 2017)等裸子植物。在槭树科 植物中, 梓叶槭的表型分化程度高于茶条槭(王丹 等, 2010)和葛萝槭等(孟超等, 2013)。种群中表型变 异小的性状更多反映了种群的生长信息(陈天翼等, 2013)。通过对梓叶械5个小种群表型性状变异系数 的分析发现, 种子厚度、果柄长度的变异程度相对 较大(表8), 这 2 个性状最能反映梓叶槭的表型变异。 种子的厚度代表了种子的饱满程度, 是影响植物能 否成功定居的主要因素(Sørensen \& Miles, 1978)。饱 满的种子更容易成功定居并繁衍后代。与表型性状 相比, 翅果千粒重和种子千粒重的变异系数较低, 但在不同种群间仍存在一定的差异: 大邑种群变异 程度最大, 说明其种实质量参差不齐, 平武种群的 翅果千粒重变异最小, 雷波种群的种子千粒重变异
最小, 说明两者分别具有相对较好的翅果和种子质 量。整体而言，与其他槭类树种(王丹等, 2010; 孟超 等, 2013)相比, 梓叶槭种实表型的变异程度略高, 但与其他物种相比则仍然较低。同时，梓叶槭种实 表型性状受气象因子影响不显著, 这可能与梓叶槭 分布范围相对较小有关。

植物表型变异主要是同种植物适应不同环境 的结果(李斌等, 2002)。本研究发现, 翅果和种子的 形态在不同种群间均存在差异, 如平武种群具有最 大的翅果, 雷波种群具有最大的种子但最短的果柄, 但两种群的翅果和种子千粒重均最大; 而都江堰种 群的种子最小, 大邑种群的翅果和着生痕最小(如 表3所示), 这可能显示了梓叶槭种群种子定居策略 的多样性(李帅峰等, 2013)。比如, 在盆地丘陵区(都 江堰)的梓叶槭种子较小、且饱满度较差, 而在盆地 边缘中山区(平武和雷波)的梓叶槭种群的种子均较 饱满。它们根据各自种群所处环境的差异, 进化选 择了不同的定居方式，如通过增加传播能力(翅果 大小)来帮助后代顺利定居。而这种差异的产生是否 与梓叶槭所生存的自然条件(低山区、湿度大、多云 雾)有关(表1), 或者与群落环境有关, 如与伴生种 间的竞争关系(许恒和刘艳红, 2018), 还有待于进一 步研究。

综上所述，梓叶槭种实表型性状在种群间和种 群内均存在显著差异, 且种群内的变异大于种群间, 
种群间分化程度相对较小。翅果千粒重是最稳定的 种实性状, 而种子厚度的变异范围最大, 是影响梓 叶棫种群繁衍的重要指标。都江堰种群的种实表型 多样性最丰富, 而平武种群则最小, 5 个种群间差异 不大。种实性状受气象因子影响不显著。就种实表 型性状而言, 雷波种群和平武种群均具有最大的种 子厚度和均一的种实质量, 可作为梓叶槭优质种质 资源的首选地。

\section{参考文献}

Chen TY, Liu ZH, Lou AR (2013) Phenotypic variation in populations of Solanum rostratum in different distribution areas in China. Chinese Journal of Plant Ecology, 37, 344-353. (in Chinese with English abstract) [陈天翌, 刘增 辉, 娄安如 (2013) 刺荳龙葵种群在中国不同分布地区 的表型变异. 植物生态学报, 37, 344-353.]

Daly C, Gibson WP, Taylor GH, Johnson GL, Pasteris P (2002) A knowledge-based approach to the statistical mapping of climate. Climate Research, 22, 99-113.

Fang WP (1981) Flora Reipbublicae Popularis Sinicae, Tomus 46. Science Press, Beijing. (in Chinese) [方文培 (1981) 中 国植物志(46卷). 科学出版社, 北京.]

Feng QH, Shi ZM, Xu ZJR, Miao N, Tang JC, Liu XL, Zhang L (2017) The study on phenotypic variations in cones and seeds of natural Cupressus chengiana populations in China. Chinese Journal of Applied Ecology, 28, 748-756. (in Chinese with English abstract) [冯秋红, 史作民, 徐峥静 茹, 缪宁, 唐敬超, 刘兴良, 张雷 (2017) 岷江柏天然种 群种实表型变异特征. 应用生态学报, 28, 748-756.]

Frankham R, Ballou JD, Briscoe DA (2002) Introduction to Conservation Genetics. Cambridge University Press, Cambridge.

Ge S, Wang MX, Chen YW (1988) Study on genetic structure of Pinus massoniana populations with isozyme. Scientia Silvae Sinicae, 24, 399-409. (in Chinese with English abstract) [葛颂, 王明庥, 陈岳武 (1988) 用同工酶研究马 尾松群体的遗传结构. 林业科学, 24, 399-409.]

Gu YJ, Lou JX, Wu YW Cao XJ (2009) Phenotypic diversity in natural populations of Picea balfouriana in Sichuan, China. Chinese Journal of Plant Ecology, 33, 291-301. (in Chinese with English abstract) [啈云杰, 罗建勋, 吴远伟, 曹小军 (2009) 川西云杉天然种群表型多样性. 植物生态学报, 33, 291-301.]

Guo Z, Zang RG (2013) Evaluation index system of endangered levels of the wild plants with tiny population in China. Scientia Silvae Sinicae, 49, 10-17. (in Chinese with English abstract) [国政, 喊润国 (2013) 中国极小种群野生植物 濒危程度评价指标体系. 林业科学, 49, 10-17.]

Hamrick JL, Godt MJW (1990) Allozyme diversity in plant species. In: Plant Population Genetics, Breeding, and Genetic Resources (eds Brown AHD, Clegg MT, Kahler AL, Wier BS), pp. 43-63. Sinauer Associates, Sunderland.
Li B, Gu WC, Lu BM (2002) A study on phenotypic diversity of seeds and cones characteristics in Pinus bungeana. Biodiversity Science, 10, 181-188. (in Chinese with English abstract) [李斌, 顾万春, 卢宝明 (2002) 白皮松天然群体 种实性状表型多样性研究. 生物多样性, 10, 181-188.]

Li S, Cai YL, Xu L, Zhao GF (2003) Morphological differentiation of samaras and seeds of Dipteronia dyeriana (Aceraceae). Acta Botanica Yunnanica, 25, 589-595. (in Chinese with English abstract) [李珊, 蔡宇良, 徐莉, 赵桂仿 (2003) 云南金钱槭果实、种子形态分化研究. 云南植物 研究, 25, 589-595.]

Li SF, Su JR, Liu WD, Lang XD, Zhang ZJ, Su L, Jiang CXZ, Yang HJ (2013) Phenotypic variations in cones and seeds of natural Pinus kesiya var. langbianensis populations in Yunnan Province, China. Chinese Journal of Plant Ecology, 37, 998-1009. (in Chinese with English abstract) [李帅锋, 苏 建荣, 刘万德, 郎学东, 张志钧, 苏磊, 贾呈銍卓, 杨华 景 (2013) 思茅松天然群体种实表型变异. 植物生态学 报, 37, 998-1009.]

Loveless MP, Hamrick JL (1984) Ecological determinant of genetic structure in plant populations. Annual Review of Ecology and Systematics, 15, 65-95.

Ma WB, Xu G, Ji HJ, Liu XL (2014) Preliminary study on germination characteristics of the rare plant Acer catalpifolium. Seed, 33(12), 87-90. (in Chinese with English abstract) [马文宝, 许戈, 姬慧娟, 刘兴良 (2014) 珍稀植 物梓叶槭种子萌发特性初步研究. 种子, 33(12), 87-90.]

Mao JF, Li Y, Liu YJ, Li H, Wang XR (2007) Cone and seed characteristic of Pinus densata and their adaptive fitness implications. Chinese Journal of Plant Ecology, 31, 291-299. (in Chinese with English abstract) [毛建丰, 李悦, 刘玉军, 刘影, 王晓茹 (2007) 高山松种实性状与生殖适 应性. 植物生态学报, 31, 291-299.]

Meng C, Zheng X, Ji ZF, Lin LL, Zhang CQ, Wang YL (2013) Phenotypic diversity of natural populations of Acer grosseri in Shanxi. Acta Botanica Boreali-Occidentalia Sinica, 33, 2232-2240. (in Chinese with English abstract) [孟超, 郑昕, 姬志峰, 林丽丽, 张翠琴, 王祎玲 (2013) 山西葛萝槭天 然种群表型多样性研究. 西北植物学报, 33, 2232-2240.]

Ren H, Zhang QM, Lu HF, Liu HX, Guo QF, Wang J, Jian SG, Bao HO (2012) Wild plant species with extremely small populations require conservation and reintroduction in China. Ambio, 41, 913-917.

Sichuan Vegetation Coordination Group (1980) Sichuan Vegetation. Sichuan People's Publishing House, Chengdu. (in Chinese) [四川植被协作组 (1980) 四川植被. 四川人民 出版社, 成都.]

Sørensen FC, Miles RS (1978) Cone and seed weight relationship in Douglas-fir from western and central Oregon. Ecology, 59, 641-644.

Wang D, Pang CH, Gao YH, Hao XJ, Wang YL (2010) Phenotypic diversity of Acer ginnala (Aceraceae) populations at different altitude. Acta Botanica Yunnanica, 32, 117-125. 
(in Chinese with English abstract) [王丹, 庞春华, 高亚卉, 郝晓杰, 王祎玲 (2010) 茶条槭不同海拔种群的表型多 样性. 云南植物研究, 32, 117-125.]

Xu H, Liu YH (2018) Relationship between diameter class structure and intraspecific and interspecific competitions of precious and endangering plant Acer catalpifolium. Acta Botanica Boreali-Occidentalia Sinica, 38, 1160-1170. (in Chinese with English abstract) [许恒, 刘艳红 (2018) 珍稀 濒危植物梓叶槭种群径级结构与种内种间竞争关系. 西 北植物学报, 38, 1160-1170.]

Yu DP, Peng QX, Li CH, Xie KP, Zhang GZ, Li SL (2008) Research on the biological characters of the seeds of Acer catalpifolium Rehd. Chinese Wild Plant Resources, 27(6), 30-64. (in Chinese with English abstract) [余道平, 彭启新, 李策宏, 谢孔平, 张国珍, 李世丽 (2008) 梓叶槭种子生 物学特性研究. 中国野生植物资源, 27(6), 30-64.]

Yu SL, Fang WW (2012) New advances in seed geography. Chinese Journal of Plant Ecology, 36, 918-922. (in Chinese with English abstract) [于顺利, 方伟伟 (2012) 种子地理 学研究的新进展. 植物生态学报, 36, 918-922.]

Zhang L, Liu SR, Sun PS, Wang TL (2011) Comparative evaluation of multiple models of the effects of climate change on the potential distribution of Pinus massoniana. Chinese Journal of Plant Ecology, 35, 1091-1105. (in Chinese with English abstract) [张雷, 刘世荣, 孙鹏森, 王 同立 (2011) 气候变化对马尾松潜在分布影响预估的多 模型比较. 植物生态学报, 35, 1091-1105.]

Zhang YY, Ma WB, Yu T, Ji HJ, Gao J, Li JQ, Gao S (2018) Study on population structure and community characteristics of Acer catalpifolium Rehd. Chinese Journal of Applied \& Environmental Biology, 24(4), 1-9. (in Chinese with English abstract) [张宇阳, 马文宝, 于涛, 姬慧娟, 高健, 李俊清, 高尚 (2018) 梓叶槭种群结构和群落特征研究. 应用与环境生物学报, 24(4), 1-9.]

(责任编委：陈又生 责任编辑：时意专) 